\section{The Pursuit of Optimal Living after Cancer through Compre- hensive Coordinated Care}

\author{
Kyle Silva1,2*, Jay Shah', Roshni Durgam¹, Michelle Stern', \\ David Prince ${ }^{1}$, Grigory Syrkin ${ }^{1}$ and Anna Lasak ${ }^{1}$
}

${ }^{1}$ Department of Physical Medicine and Rehabilitation, Montefiore Medical Center, Albert Einstein College of Medicine, University in New York City, New York, USA

${ }^{2}$ Jacobi Medical Center, Hospital in New York City, New York, USA

\begin{abstract}
There are 13.7 million cancer survivors in the US today. Unfortunately, many suffer from post-cancer treatment sequelae which often go un-treated. Cancer Rehabilitation is an upcoming sub-specialty of Physical Medicine and Rehabilitation that focuses on helping to reduce the morbidity for cancer survivors suffering from post-cancer treatment complications. The primary goal of this article is to raise awareness amongst the medical community about the impact of cancer rehabilitation on cancer survivorship. Cancer rehabilitation offers an improved quality of life through the treatment of musculoskeletal, neuromuscular, and functional complications experienced by cancer survivors. With the increased awareness of rehabilitative treatment options amongst the medical community, more cancer survivors will be able to live an optimal, cancer-free life. Keywords: Cancer; Rehabilitation; Survivor
\end{abstract}

\section{Manuscript}

According to 2013 American Cancer Society (ACS) statistics, there are approximately 13.7 million American cancer survivors and the number is growing. Today it is estimated that $65 \%$ of those diagnosed with cancer will live at least 5 years [1]. Many cancer survivors face multiple challenges with returning to their pre-morbid activities of daily living as a result of post-cancer treatment sequelae. The majority of impairments that cancer survivors suffer from can be treated and managed with rehabilitative interventions.

Cancer rehabilitation has been defined by an article by Cromes as assisting cancer survivors to obtain maximum physical, social,

*Corresponding author: Kyle Silva, Department of Physical Medicine and Rehabilitation, Montefiore Medical Center, Albert Einstein College of Medicine, University in New York City, 309 W47 $7^{\text {th }}$ St. Apt 1FW NY, New York 10036, USA, Tel: +1 5085421444; E-mail: drkylesilva@gmail.com

Citation: Silva K, Shah J, Durgam R, Stern M, Prince D, et al. (2015) The Pursuit of Optimal Living after Cancer through Comprehensive Coordinated Care. J Phys Med Rehabil Disabil 1: 004.

Received: August 04, 2015; Accepted: September 04, 2015; Published: September 25, 2015 psychological, and vocational functioning through the use of an interdisciplinary team approach [2]. Cancer rehabilitation is underutilized throughout the United States. This occurs due to a lack of provider education and understanding of the benefits that a cancer rehabilitation professional can offer cancer survivors. In a 2008 study performed by Cheville at the Mayo Clinic along with contributions from Memorial Sloan-Kettering Cancer Center, 92\% of 163 patients with metastatic breast cancer were found to have at least one physical impairment for which referral to a cancer rehabilitation specialist would have been appropriate. However, the study revealed that only $30 \%$ of individuals with impairments requiring intervention by a cancer rehabilitation specialist received referrals to have these impairments further evaluated [3].

Underutilization of cancer rehabilitation is also an international problem. In Norway, researchers looked at 1,325 patients diagnosed with cancer from 2005-2006 and determined that $63 \%$ of these patients had unmet physical and psychosocial needs [4]. A retrospective longitudinal cohort study was performed by the National Health Insurance research database in Taiwan which showed that only $39.6 \%$ of 632 patients with breast cancer received rehabilitative services [5]. Ultimately, the need for cancer rehabilitation is evident worldwide, however these needs are not being met.

The importance of the role played by Physiatrists in ensuring comprehensive care for cancer survivors cannot be overemphasized. Physiatrists specializing in cancer rehabilitation help to coordinate care amongst primary care physicians, medical oncologists, radiation oncologists, surgical specialists, and psychiatrists [6]. In addition, Physiatrists are adept at evaluating functional impairment and determining what type of intervention may be needed. They are capable of treating pain by utilizing therapy services, providing pharmacologic management, and through interventional procedures. Furthermore, many physiatrists are adept at acupuncture and lymphedema services, both of which can be utilized to treat post-cancer sequelae.

Since physical impairments limit the patient's ability to function optimally and are associated with poor overall survival, early identification and intervention is of the utmost importance [7]. As the number of cancer survivors continues to increase so too will the need for improved recognition of functional impairments and access to rehabilitative care. This can only be achieved through the increased awareness of primary care physicians about post-cancer treatment sequelae and rehabilitative interventions available to address these specialized needs.

The specialized needs of cancer survivors are many, ranging from a variety of impairments such as: anatomic, physiologic, and psychological impairments and how they manifest to produce disability and handicap. Cancer rehabilitation focuses on the restoration of functional losses from direct or indirect causes during the treatment of cancer. Direct causes are related to impairments produced from cancerous lesions, i.e., pathologic fractures, disruption of normal biomechanics, pain and fatigue. While indirect causes can include complications of radiation, chemotherapy, and 
surgical intervention such as radiation fibrosis, lymphedema, and disruption of normal biomechanics after surgical intervention. Ultimately, the goal of cancer rehabilitation is to offer an improved quality of life by treating the musculoskeletal, neuromuscular, and functional complications experienced by cancer survivors (Table 1).

\begin{tabular}{|c|}
\hline Physical Impairments \\
\hline Impaired range of motion \\
\hline Gait and balance dysfunction \\
\hline Deconditioning \\
\hline Decreased cardiovascular capacity \\
\hline Dysphagia \\
\hline Dysarthria \\
\hline Lymphedema \\
\hline Radiation fibrosis \\
\hline Post-surgical scar adhesion management \\
\hline Myelopathy \\
\hline Cardiomyopathy \\
\hline Bowel/Bladder dysfunction \\
\hline Cervical dystonia \\
\hline Trismus \\
\hline Functional impairments \\
\hline Inability to perform activities of daily living \\
\hline Inability to return to prior activity level (work, home, recreational, social) \\
\hline Adaptive equipment needs \\
\hline Pain/Fatigue management \\
\hline Neuropathic pain \\
\hline Biomechanical pain \\
\hline Musculoskeletal pain \\
\hline Phantom pain \\
\hline Cancer related fatigue syndrome \\
\hline Psychosocial concerns \\
\hline Dysfigurement \\
\hline Economic burden \\
\hline
\end{tabular}

Table 1: Post-cancer treatment sequelae addressed by rehabilitation specialists include, but are not limited to the following table.

There is strong evidence in the medical literature that supports the efficacy of cancer rehabilitation in optimizing survivor function (Table 2). A meta-analysis performed by Mishra et al., analyzed the effects of exercise on health-related quality of life. Their data suggests that exercise can improve body image, self-esteem, emotional well-being, sexuality, social functioning, and fatigue, ultimately allowing for an improved quality of life [8]. Similarly, a meta-analysis performed by Cramp et al., determined that patients suffering from prostate or breast cancer-associated fatigue benefited from resistance training both during and post-treatment [9]. Cho and researchers looked at the efficacy of comprehensive rehabilitation on shoulder Range of Motion (ROM) after mastectomy. Their results showed an increase in ROM from $81.4 \%$ to $92.9 \%$ improvement over baseline ROM in patients receiving rehabilitation versus no observable change in their control group [10]. A meta-analysis looking at the efficacy of exercise in improving cancer related fatigue confirmed that there is a moderate improvement in fatigue at a statistically significant level [11]. Due to ample evidence-based research in the literature about the efficacy of rehabilitative care, referral of all cancer survivors to cancer rehabilitation specialists is quickly becoming standard of care.

\begin{tabular}{|c|}
\hline Endurance \\
\hline Balance and coordination \\
\hline Cardiovascular conditioning \\
\hline Muscular strength and range of motion \\
\hline Bone mass density \\
\hline Pain control \\
\hline Fatigue \\
\hline Lymphedema and immune functioning \\
\hline Functional ability \\
\hline Quality of life \\
\hline
\end{tabular}

Table 2: Evidence based results of cancer rehabilitation improves but is not limited to the following: [12-18].

The 2012 guidelines released by the American College of Surgeons Commission on Cancer (COC) include rehabilitative services as a recognized evidence-based standard of care for cancer survivors [12]. To ensure comprehensive care for cancer survivors, the COC recommends that all cancer treatment facilities offer access to rehabilitative services either within their facility or through referral to an outside provider [13]. Ultimately, the goal of the COC is to provide cancer survivors with a comprehensive survivorship plan to address all cancer-related complications.

The cost of treating cancer today continues to increase. From a monetary standpoint, early rehabilitative intervention focused on early intervention may decrease the overall cost of treating cancer and its complications. Too often, cancer survivors are receiving rehabilitative services once they are severely debilitated. This results in increased re-hospitalization rates and increased absences from work. Through early intervention by cancer rehabilitation specialists who treat patient function, dysfunction can be identified and treated before it becomes a significant disability for the patient.

There is opportunity for improvement regarding the medical care that is offered to cancer survivors in the US today. Although the future of cancer rehabilitation and its implications for cancer survivorship is promising, there is still a great deal of provider education that must occur for cancer survivors to benefit from state of the art cancer rehabilitation. A major barrier which persists is the lack of knowledge by both patients and physicians about cancer rehabilitative services, thus limiting access to care. As awareness amongst health care providers continues to grow, so will the quality of life for all cancer survivors. Ultimately, additional accessibility to rehabilitative treatment will lead to more opportunities to prevent or relieve the suffering endured by cancer survivors.

\section{References}

1. American Cancer Society (2013) Palliative care research, USA.

2. Cromes GF (1978) Implementation of interdisciplinary cancer rehabilitation. Rehabil Counseling Bull 21: 230-237.

3. Cheville AL, Troxel AB, Basford JR, Kornblith AB (2008) Prevalence and treatment patterns of physical impairments in patients with metastatic breast cancer. J Clin Oncol 26: 2621-2629.

4. Thorsen L, Gjerset GM, Loge JH, Kiserud CE, Skovlund E, et al. (2011) Cancer patients' needs for rehabilitation services. Acta Oncol 50: 212-222. 
5. Lin YH, Pan PJ (2012) The use of rehabilitation among patients with breast cancer: a retrospective longitudinal cohort study. BMC Health Serv Res 12 282.

6. Bodenheimer T, Lo B, Casalino L (1999) Primary care physicians should be coordinators, not gatekeepers. JAMA 281: 2045-2049.

7. Gerber LH, Stout NL, Schmitz KH, Stricker CT (2012) Integrating a prospective surveillance model for rehabilitation into breast cancer survivorship care. Cancer 118: 2201-2206

8. Mishra SI, Scherer RW, Geigle PM, Berlanstein DR, Topaloglu O, et al. (2015) Exercise interventions on health-related quality of life for cancer survivors (Review). The Cochraine collaboration, John Wiley \& Sons, USA

9. Minton O, Berger A, Barsevick A, Cramp F, Goedendorp M, et al. (2013) Cancer-related fatigue and its impact on functioning. Cancer 119: 2124-2130.

10. Cho OH, Yoo YS, Kim NC (2006) Efficacy of comprehensive group rehabilitation for women with early breast cancer in South Korea. Nurs Health Sci 8: $140-146$.

11. Brown JC, Huedo-Medina TB, Pescatello LS, Pescatello SM, Ferrer RA, et al. (2011) Efficacy of exercise interventions in modulating cancer-related fatigue among adult cancer survivors: a meta-analysis. Cancer Epidemiol Biomarkers Prev 20: 123-133.
12. Oncology Rehab Partners (2015) Survivorship Training and Rehab Program®. Oncology Rehab Partners, Marlborough, MA, USA.

13. Greene FL, Witmer DD, Edge SB, Winchester DP, Dickerson D, et al. (2012) Cancer Program Standards 2012: Ensuring Patient-Centered Care. Commission on Cancer V1, American College of Surgeons 1: 31-38.

14. De Backer IC, Vreugdenhil G, Nijziel MR, Kester AD, van Breda E, et al. (2008) Long-term follow-up after cancer rehabilitation using high-intensity resistance training: persistent improvement of physical performance and quality of life. Br J Cancer 99: 30-36.

15. Hojan K, Milecki P, Leszczyński P (2013) The impact of aerobic exercises on bone mineral density in breast cancer women during endocrine therapy. Pol Orthop Traumatol 78: 47-51.

16. Dimeo FC, Tilmann MH, Bertz H, Kanz L, Mertelsmann R, et al. (1997) Aerobic exercise in the rehabilitation of cancer patients after high dose chemotherapy and autologous peripheral stem cell transplantation. Cancer 79: $1717-1722$

17. Torres Lacomba M, Yuste Sánchez MJ, Zapico Goñi A, Prieto Merino D, Mayoral del Moral O, et al. (2010) Effectiveness of early physiotherapy to prevent lymphoedema after surgery for breast cancer: randomised, single blinded, clinical trial. BMJ 340: 5396.

18. Marciniak CM, Sliwa JA, Spill G, Heinemann AW, Semik PE (1996) Functional outcome following rehabilitation of the cancer patient. Arch Phys Med Rehabil 77: 54-57. 